\title{
DE-JITTER BUFFER ROLE IN IMPROVING VOIP CONNECTION QUALITY - EXAMPLES FROM PRACTICE
}

\author{
Aleksandar Lebl', \\ Mladen Mileusnić1, \\ Dragan Mitić', \\ Žarko Markov', \\ Branislav Pavić ${ }^{1}$
}

${ }^{1}$ IRITEL a.d., Belgrade, Batajnički put 23, Serbia

Correspondence:

Aleksandar Lebl

e-mail:

lebl@iritel.com

\begin{abstract}
:
This paper presents the way the optimum de-jitter buffer delay is determined to achieve maximum VoIP connection quality. This delay estimation is based on presentation of 1-CDF (1- cumulative distribution function) characteristics of packet delay distribution in the field of equiquality lines "delay-packet loss“ for applied coder (compressor). The results are based on a real measurement of end-to-end delay for different links in Internet and on coder (compressor) send side delay. It is proved that five-sixths connections have high or medium connection quality and that the corresponding de-jitter buffer delay is relatively small, while in about $5 \%$ of connections satisfactory connection quality cannot be achieved.
\end{abstract}

Keywords:

VoIP connection quality, E-model, equiquality lines „delay-packet loss“, de-jitter buffer.

\section{INTRODUCTION}

Today Internet has become global world network. All kinds of people communication are realized over Internet. Among these communications, voice (or VoIP) communication is still one of the most important ones. That's why the question is whether satisfactory quality can be achieved in these voice connections and how this quality can be improved as much as possible. This paper analyzes voice quality, which can be expected according to real measured characteristics of Internet links, [1], [2], and what de-jitter buffer characteristics have to be realized at the receiving connection sides to improve connection quality as much as possible. In section I main specifications of E-model (intended for analysis of VoIP connection quality) are emphasized. Also, here the role of de- jitter buffer is described. In section II model of VoIP connection is presented and the contribution of each element in this model to packet delay is mentioned. Section III deals with distribution of packet end-to end delay, according to performed measurements. Section IV presents achievable connection quality and estimates optimum value of de-jitter buffer delay. Finally, section $\mathrm{V}$ is the conclusion. 


\section{E-MODEL AND WHY DE-JITTER BUFFER DELAY IS IMPORTANT}

E-model is computational method for estimation of VoIP connection quality. It joins influence of many heterogeneous factors into one unique quality estimation - rating factor $(R)$.

The value of $\mathrm{R}$ is between 0 and 100 , but, practically, the best quality is $R 0=94$ for local ISDN connection. On the other side, values $R<50$ correspond to unsatisfactory voice connection quality. For $50<R<70$ connection quality is low and for $R>70$ it is high or medium, [3], [4].

There are several factors, which decrease maximum VoIP connection quality $(R 0)$. According to equation (71) from [3], these are simultaneous impairment factor $\left(I_{s}\right)$, delay impairment factor $\left(I_{d}\right)$ and equipment impairment factor $\left(I_{e}\right)$, or, better said, equipment effective impairment factor $\left(I_{\text {eefff }}\right)$. In this equation advantage factor $(A)$ is a psychological element, which increases estimated voice quality. For the analysis in this paper two important components, which decrease VoIP connection quality are packet (transmission) delay (expressed as one of components included in factor $I_{d}$ ) and packet loss (included in factor $\left.I_{\text {e-eff }}\right)$. As packet delay and packet loss are greater, voice connection quality is worse, i.e. value of $R$ is smaller. The effects of packet delay and packet loss are mutually different. It means that, when considering packet loss, we can improve $R$ by implementation of greater buffers at the receiving side, thus „catching “ packets with greater delay. But, it means that it is necessary to increase delay of all packets to the delay of a packet with the greatest acceptable delay. Packet delay increasing leads, on the other side, to $R$ deterioration. If we, on the other hand, implement smaller buffers, packet delay is smaller and connection quality is better if we consider delay. But, in the same time, packet loss is greater and $R$ is smaller if we consider this loss. The consequence of this qualitative analysis is that there should be some optimum pair of values of packet delay and loss, where VoIP connection quality is greatest. The role of de-jitter buffer is to eliminate (not accept) all packets with greater delay than the optimum one.

\section{MODEL OF VOIP CONNECTION AND FACTORS OF VOICE CONNECTION QUALITY DECREASE}

A model of one VoIP connection between two telephone users (TA and TB) is presented in Figure 1, [5]. The elements, included in connection realization can be divided in three groups: elements on the sending side, elements on the link between two users and elements on the receiving side.

Gateways (GWA and GWB) are situated on the sending and receiving side of the connection. On the sending side in GWA voice connections packets are prepared for transmission. Here the most important activities are voice signal compression (coding), packetization, implementation of outgoing signal buffer and voice activity detection (VAD). On the receiving side in GWB voice connection packets are decompressed (decoded), depacketized and delay of all received packets is equated by de-jitter buffer implementation.

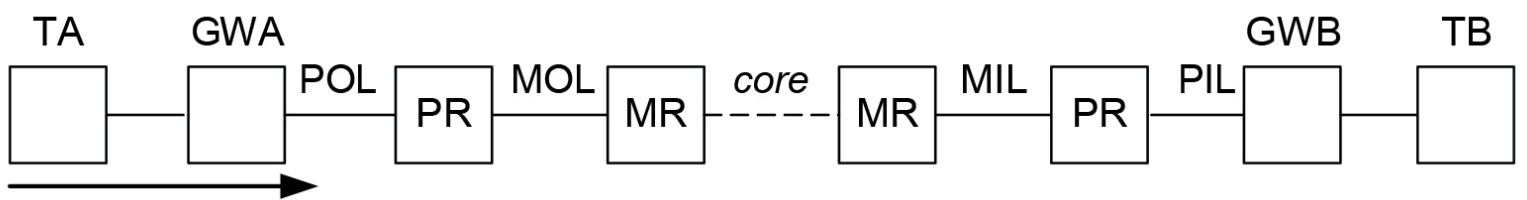

TA, TB - telephone; GWA, GWB - gateway; POL, PIL - peripheral outgoing an incoming link; MOL, MIL magistral outgoing an incoming link; PR, MR - peripheral, magistral router; core - magistral network

Fig. 1. A model of VoIP connection.

The other elements in Figure 1 form a route between GWA and GWB, i.e. between users (connection participants). The elements of this route are routers (peripheral routers - PR and magistral routers - MR) and links (peripheral outgoing and incoming links - POL and PIL, and magistral outgoing and incoming links - MOL and MIL).
All elements presented in Figure 1, which are included in packet transmission, contribute to the decrease of voice connection quality. This quality decrease is, first of all, the result of packet delay. Each element in Figure 1 increases (more or less) total packet end-to-end delay. Packet loss is, also, important factor, which decreases voice connection 
quality. But, in modern networks, with the implementation of good quality optical links and high-speed signal processing, as well as improved protocols, importance of packet loss becomes less significant.

Delay of the elements on a route between GWA and GWB is called end-to-end delay and the results of its measurement can be found in literature, as, for example, in [1], [2]. The signal processing in GWA and GWB increases voice signal delay between users over the value of end-to end delay. Signal delay on the sending side is dominant comparing to delay on the receiving side. This delay (called send side delay - $t_{s d}$ ) on the sending side is necessary to perform coding (compression), packetization and to achieve correct timing relations between transmitted packets. The values of $t_{s d}$ for different coder (compressor) types are presented in [6], and the values for coders (compressors), which are analyzed in this paper, are presented in Table I, [7]. The analyzed coders (compressors) are G.711 without packet loss concealment (PLC), G.711 with PLC, G.729 with VAD (G.729+VAD) and G.723.1. The values of tsd are added to end-to-end delay.

\begin{tabular}{cc}
\hline Coder (compressor) & $\boldsymbol{t}_{\boldsymbol{s d}}(\mathbf{m s})$ \\
\hline G.711 & 0.375 \\
\hline G.723.1 & 97.5 \\
\hline G.729 & 35
\end{tabular}

Table 1. Maximum values of send side delay.

\section{DISTRIBUTION OF PACKET DELAY}

The results of our analysis of optimum de-jitter buffer delay are based on comprehensive delay measurements between Internet network nodes. According to the results of measurements, presented in [1], [2], all paths between test- boxes, situated all over the world, can be classified in five classes, when packet delay is considered. The first four of them are categorized according to their specific shape of delay probability density function (PDF), while the fifth one can't be categorized (the measured delay traces mainly occurred only once and they do not fit to any of the first four traces).

The first four end-to-end delay distribution classes are presented in figures $2-5$. These distribution classes are named according to the delay distribution PDF shape: class A (or Gamma-like with heavy tail), class B (or Gamma-like with Gaussian or triangle lob), class C (or Two gamma-like distributions) and class $\mathrm{D}$ (or Many peaks). Class A is the most frequent distribution class (84\% of traces), while the other three classes are significantly less frequent (class B distribution is noticed in $6.3 \%$ of cases, class $\mathrm{C}$ in $2.8 \%$ of cases and class $\mathrm{D}$ in $5 \%$ of cases). For our analysis the most important elements for each of these distributions are time 95\% (it is expected that 95\% of packets on the link have smaller delay), time $99 \%$ (it is expected that $99 \%$ of packets on the link have smaller delay) and maximum delay. The concrete values for the presented distributions are emphasized in figures 2-5. On the basis of these values and the plotted PDF characteristics, it is possible to determine 1-CDF distribution characteristic for the last $5 \%$ of packet delay PDF. The optimum value of de-jitter buffer delay is always found in these last $5 \%$ of $1-\mathrm{CDF}$ characteristic.

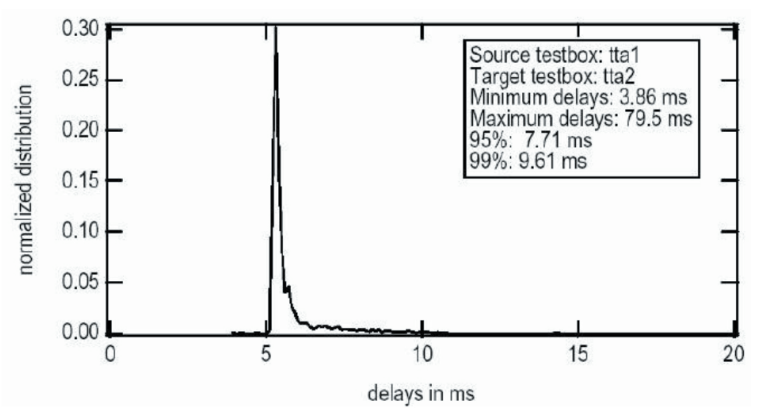

Fig. 2. Class A PDF of packet end-to-end delay distribution-gamma-like shape with heavy tail, taken from [1], [2]

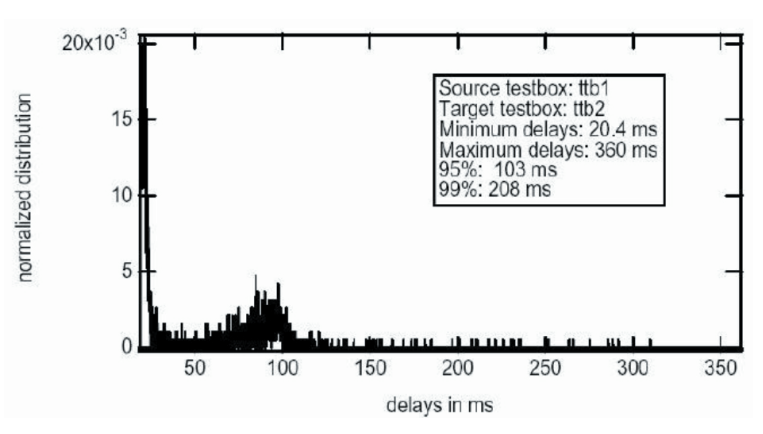

Fig. 3. Class B PDF of packet end-to-end delay distribution-gamma-like with Gaussian or triangular lob, taken from [1], [2].

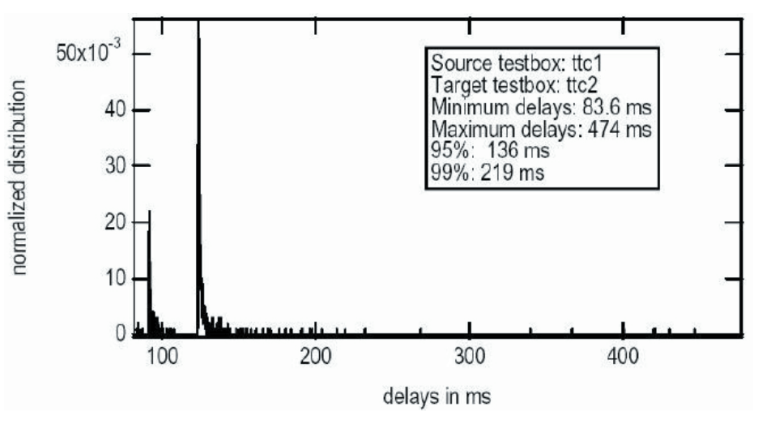

Fig. 4. Class $\mathrm{C} P D F$ of packet end-to-end delay distribution-two gamma-like distributions, taken from [1], [2]. 


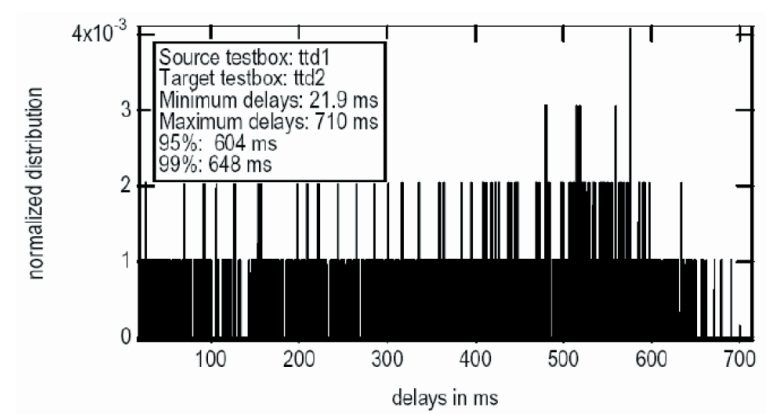

Fig. 5. Class D PDF of packet end-to-end delay distribution - many peaks, taken from [1], [2].

\section{ESTIMATION OF VOIP CONNECTION QUALITY}

The estimation of maximum VoIP connection quality may be performed in the field of equiquality lines "delaypacket loss". The way how these, originally developed, characteristics are constructed is presented in [8], [9].

[8], [9] also describe how cumulative distribution function $(\mathrm{CDF})$ characteristics of packet delay are constructed and how 1-CDF characteristics, plotted in a field of equiquality lines, are used to determine maximum voice connection quality. These characteristics are also used to determine optimum de-jitter buffer delay.

Modified characteristics 1-CDF for four classes of packet delay distribution (classes A, B, C and D) are presented in the field of equiquality lines in figures 6-18. The modification of original 1-CDF characteristics supposes addition of $t_{s d^{\prime}}$. It means that original characteristics are shifted to the right for $t_{s d^{d}}$. This shift can be noticed in the case of G.729+VAD and G.723.1 compressors, while in the case of G.711 it is negligible. Characteristic 1-CDF in figures 6-18 is a trend line of real 1-CDF characteristic, obtained according to packet delay PDFs in figures 2-5.

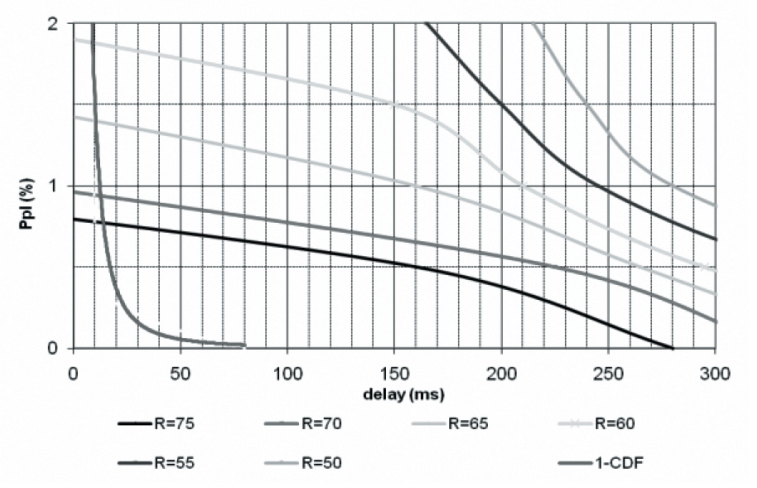

Fig. 6. VoIP connection quality for class A delay packet distribution when G.711 coder without PLC is implemented.

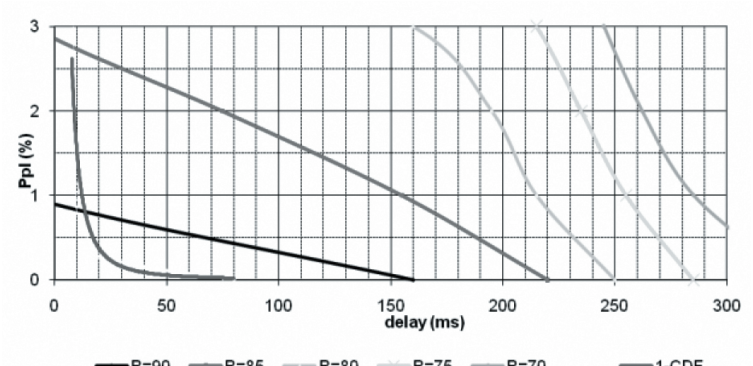

- $\mathrm{R}=90 \longrightarrow \mathrm{R}=85-\mathrm{R}=80 \longrightarrow \mathrm{R}=75-\mathrm{R}=70 \quad$ - $\mathrm{CDF}$

Fig. 7. VoIP connection quality for class A delay packet distribution when G.711 coder with PLC is implemented.

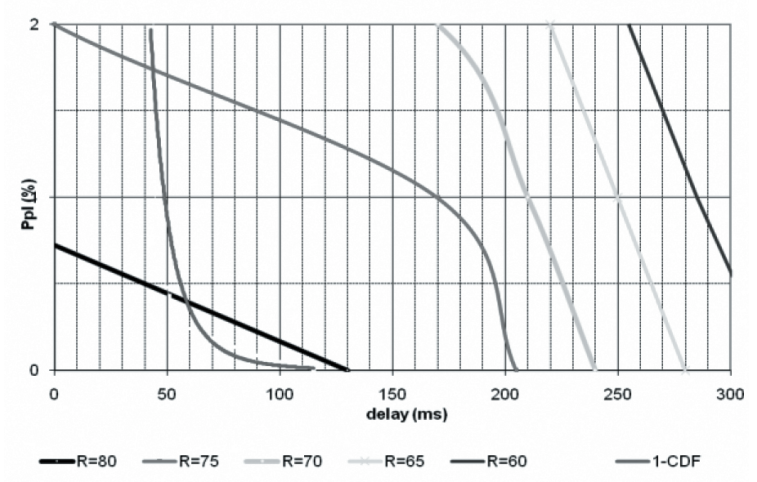

Fig. 8. VoIP connection quality for class A delay packet distribution when G.729+VAD compressor is implemented.

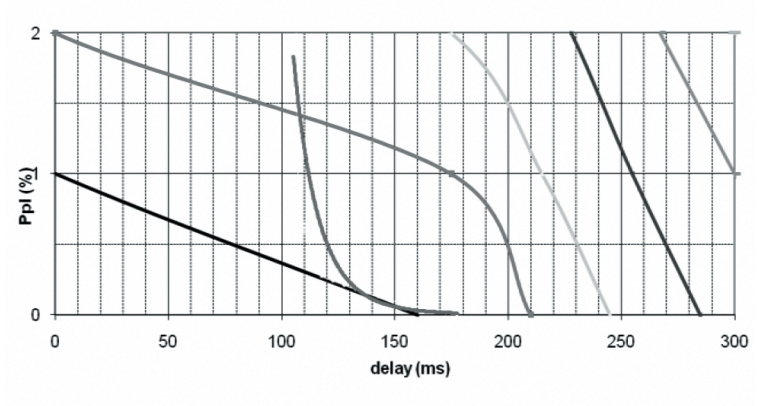

- $\mathrm{R}=75 \longrightarrow \mathrm{R}=70-\mathrm{R}=65-\mathrm{R}=60-\mathrm{R}=55-\mathrm{R}=50 \quad$ - 1 -CDF

Fig. 9. VoIP connection quality for class A delay packet distribution whenG.723.1 compressor is implemented.

Figures 6-9 can be used to determine voice connection quality and optimum de-jitter buffer delay for class A packet distribution. Figure 6 corresponds to the case of G.711 coder without PLC, Figure 7 to G.711 coder with PLC, Figure 8 to G.729+VAD compressor and Figure 9 to G.723.1 compressor. Optimum de-jitter buffer delay is the one, where voice quality is maximum. 
Figures 10-17 are used to determine voice connection quality and optimum de-jitter buffer delay for class B (figures 10-13) and for class $C$ packet distributions (figures 14-17). Figures 10 and 14 are for G.711 coder without PLC, figures 11 and 15 for G.711 with PLC, figures 12 and 16 for G.729+VAD compressor and figures 13 and 17 for G.723.1 compressor.
In the case of class $\mathrm{D}$ distribution acceptable voice quality can't be achieved. It can be concluded from Figure 18 that, even in the case of implementation of G.711 coder with PLC, which always gives the best voice quality, the connection rating factor is $R<50$.

\begin{tabular}{|c|c|c|c|}
\hline class & Coder (compressor) & tDB (ms) & $\mathbf{R}$ \\
\hline \multirow{4}{*}{ A } & G.711 without PLC & 40 & $<80$ \\
\hline & G.711 with PLC & 40 & 92 \\
\hline & G.729+VAD & 80 & 81.5 \\
\hline & G.723.1 & 140 & 75 \\
\hline \multirow{4}{*}{ B } & G.711 without PLC & 260 & 70 \\
\hline & G.711 with PLC & 140 & 82 \\
\hline & G.729+VAD & 180 & 68 \\
\hline & G.723.1 & 220 & 56.5 \\
\hline \multirow{4}{*}{$\mathrm{C}$} & G.711 without PLC & 150 & 57.5 \\
\hline & G.711 with PLC & 110 & 81.5 \\
\hline & G.729+VAD & 140 & 66 \\
\hline & G.723.1 & 200 & 53.5 \\
\hline \multirow{4}{*}{$\mathrm{D}$} & G.711 without PLC & & $<50$ \\
\hline & G.711 with PLC & & $<50$ \\
\hline & G.729+VAD & & $<50$ \\
\hline & G.723.1 & & $<50$ \\
\hline
\end{tabular}

Table 2. Optimum de-jitter buffer delay and corresponding connection rating factor.

Optimum de-jitter buffer delay $\left(t_{D B}\right)$ and corresponding connection rating factor $(R)$ for all packet distributions and coder (compressor) types from figures 6-18, are presented in table II. The values of $t_{D B}$ are obtained as the difference of values, estimated from figures 6-18, and the minimum packet delay, emphasized in figures 2-5.

When considering values from table II, it can be concluded that high or medium VoIP connection quality can be achieved for class A packet delay distributions, regardless of the type coder (compressor), and this is the situation in about five-sixths of connection routes. Optimum de-jitter buffer time in these cases is not great, meaning that there is no need for very great memory capacity to realize de-jitter buffer. For class D packet delay distribution ( $5 \%$ of routes) it is not possible to achieve the threshold of satisfactory voice quality $R=50$.

Between two mentioned packet groups are classes $B$ and C. A higher value of $\mathrm{R}$ can be achieved for the class $\mathrm{B}$, than for class C. However, the delay (and, therefore, the required memory capacity) is higher in the case of class B. It is characteristic for class $C$ packet delay distribution that the call quality is even worse significantly if G.711 coder without PLC is applied than if G.729+VAD is implemented. For the same class C, the quality of G.711without PLC connection is not much better (about $\Delta R=4$ units) comparing to G.723.1 compressor connection. In the case of class $\mathrm{B}$ and class $\mathrm{C}$ distributions, only implementation of the best coder G.711 with PLC guarantees connection quality, which is not low $(R>70)$. 


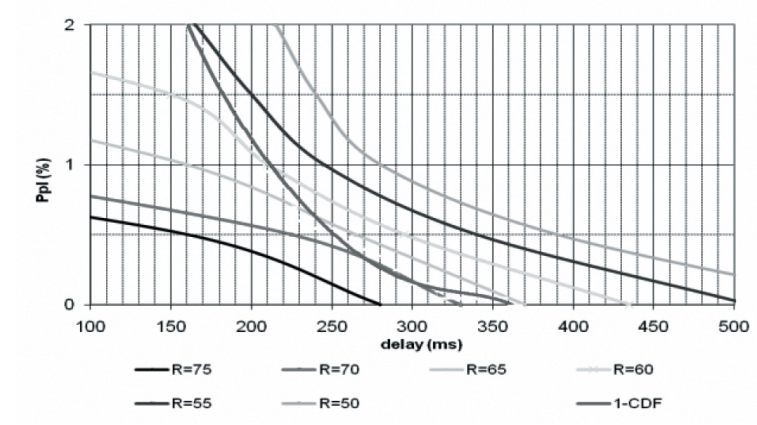

Fig. 10. VoIP connection quality for class B delay packet distribution when G.711 coder without PLC is implemented.

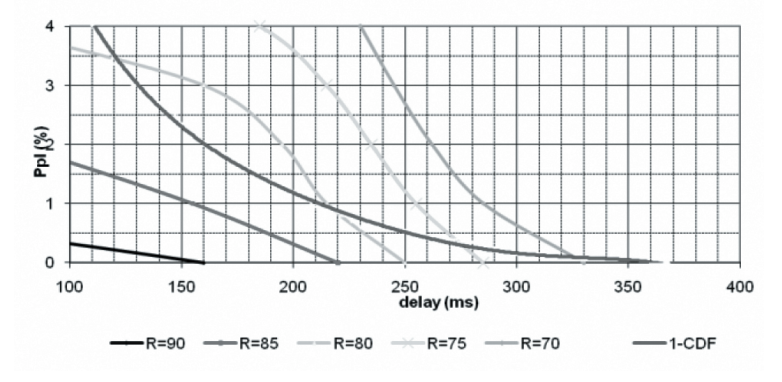

Fig. 11. VoIP connection quality for class B delay packet distribution when G.711 coder with PLC is implemented.

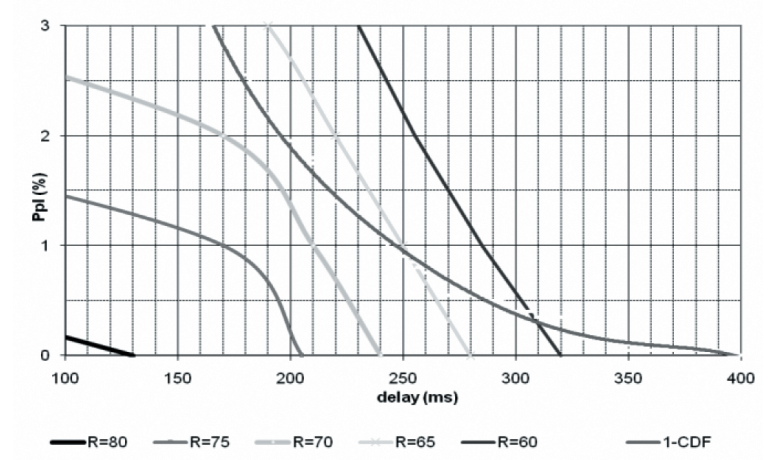

Fig. 12. VoIP connection quality for class B delay packet distribution when G.729+VAD compressor is implemented.

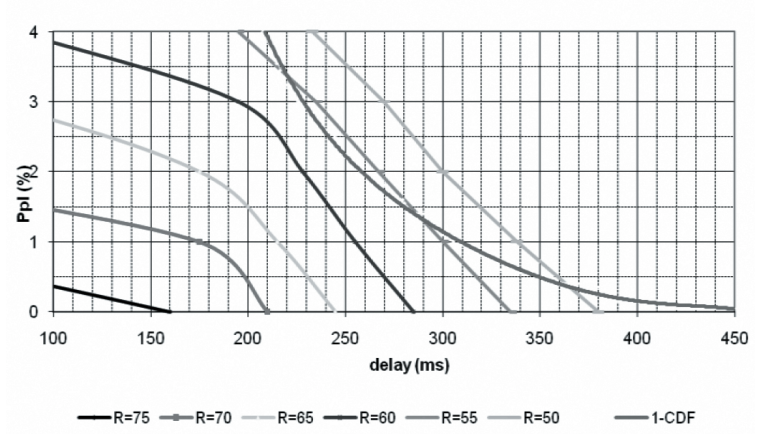

Fig. 13. VoIP connection quality for class B delay packet distribution when G.723.1 compressor is implemented.

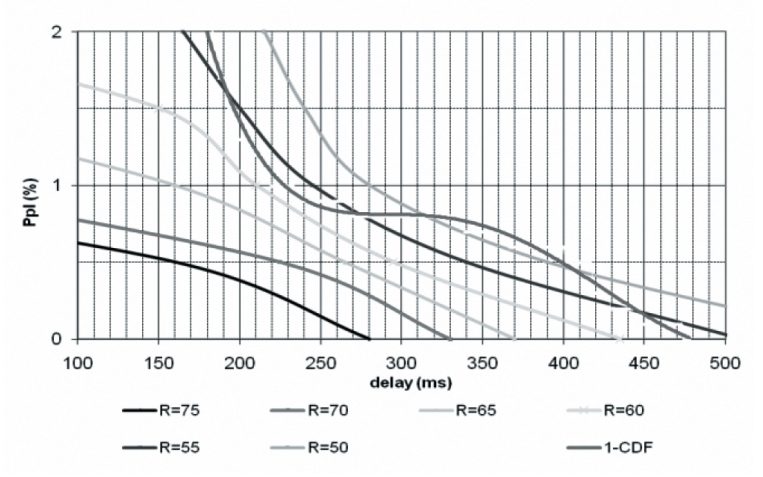

Fig. 14. VoIP connection quality for class $C$ delay packet distribution when G.711 coder without PLC is implemented.

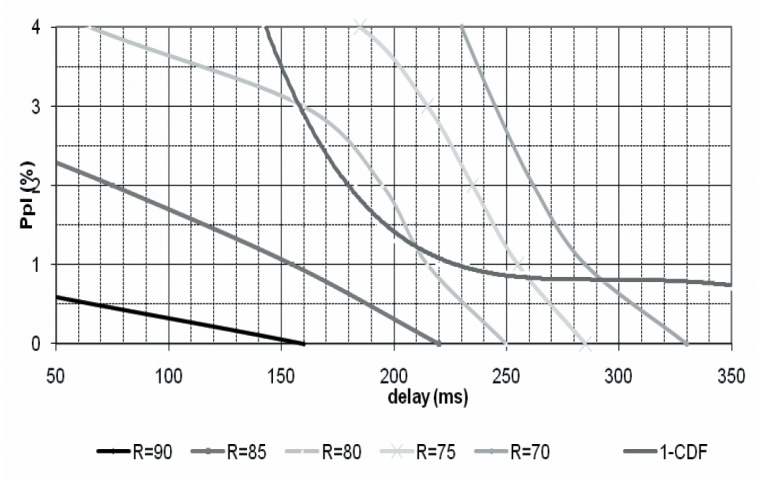

Fig. 15. VoIP connection quality for class $\mathrm{C}$ delay packet distribution when G.711 coder with PLC is implemented. 


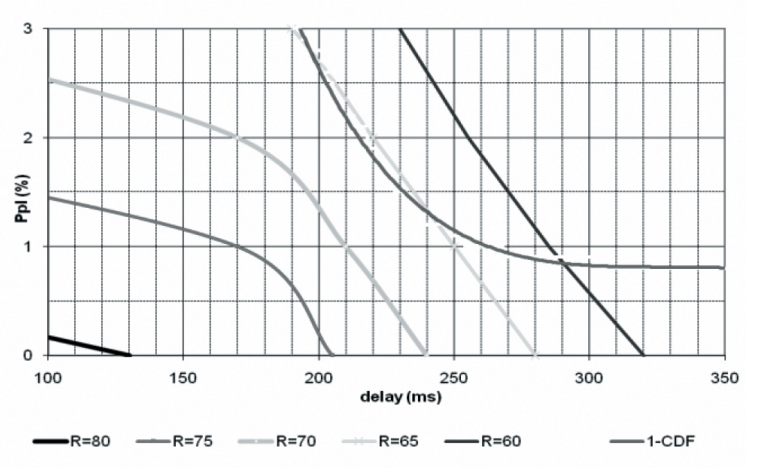

Fig. 16. VoIP connection quality for class $C$ delay packet distribution when G.729+VAD compressor is implemented.

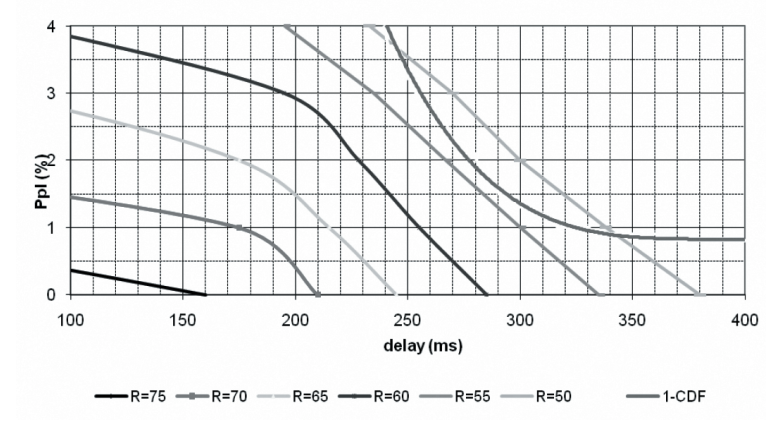

Fig. 17. VoIP connection quality for class C delay packet distribution when G.723.1 compressor is implemented.

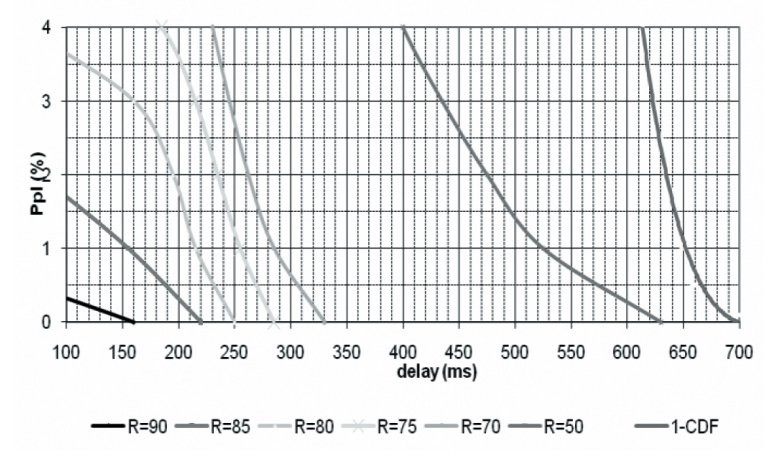

Fig. 18. VoIP connection quality for class D delay packet distribution when G.711 coder with PLC is implemented.

\section{CONCLUSION}

In this paper the measured PDFs of end-to-end delay in packet transmission over Internet, together with send side delay in packets prepared at the sending side, are used as the starting point to determine 1-CDF total packet transmission delay characteristics. These 1-CDF characteristics are then presented in the field of equiquality lines "delay-packet loss“, which are specific for each coder (compressor). From such combined characteristics it is possible to choose optimum de- jitter buffer delay to obtain maximum VoIP connection quality.

As the result of analysis, it can be concluded that great majority of connections (five-sixths with class A PDF distribution) have high or medium voice quality $(R>70)$, regardless of coder (compressor) type. Necessary de-jitter buffer delay is small in these cases, so there is no need for a great memory capacity. Further about $10 \%$ of connections have worse quality, which is in the area of low connection quality $(50<R<70)$ for all coder (compressor) types, except for G.711 coder with PLC. In these cases it is advisable to use the best coder type. On the last group of connections (more than 5\% of links in class D) connection quality is unsatisfactory, regardless of coder (compressor) type.

\section{ACKNOLEDGMENT}

The paper is realized within the project TR32051, which is financed by the Ministry of Education, Science and Technological Development of Republic of Serbia.

\section{DEDICATION}

Paper is devoted to the fiftieth anniversary of Institute IRITEL from Belgrade.

\section{REFERENCES}

[1] M. Karakaş, "Determination of Network Delay Distribution over the Internet," master thesis. The Middle East Technical University, The Graduate School of Natural and Applied Sciences, Ankara, 2003.

[2] C. J. Bovy, H. T. Mertodimedjo, G. Hooghiemstra, H. Uijterwaal, and P. Van Mieghem, "Analysis of End-to-end Delay Measurements in Internet, "In The Third Passive \& Active Measurement Workshop, Fort Collins, Colorado, March 2002.

[3] ITU-T Recommendation G.107, "The E-model, a computational model for use in transmission planning," 2015.

[4] Telecommunications industry association, "Telecommunications - IP Telephony Equipment Voice Quality Recommendations for IP Telephony: TSB-116-A,“ TIA Telecommunications Systems Bulletin, 2006. 
[5] Ž. Markov, "Modern Telephone Technics (600 Questions and Answers)," IRITEL, in Serbian, 2005.

[6] ITU-T Recommendation G.114, "One-way transmission time," 2003.

[7] A. Lebl, D. Mitić, P. Petrović, V. Matić, M. Mileusnić, and Ž. Markov, "Implementation of the Lines of Equal Quality „Delay-Echo-Packet Loss“ in Internet Voice Connection Planning," In XV International Scientific-Professional Symposium Infoteh-Jahorina, March 2016, pp. 284-289.
[8] M. Mileusnić, A. Lebl, D. Mitić, and Ž. Markov, "About Delay Loss Equiquality Characteristics in Packet Telephony, “ Automatika, vol. 55, no. 1, April 2014., pp. 64-68., DOI: 10.7305/automatika.2014.01.231

[9] A. Lebl, V. Marinković-Nedelicki, M. Mileusnić, D. Mitić, V. Matić, Ž. Markov, and I. Marjanović, "Optimal De-Jitter Buffer Dimensioning as the Function of VoIP Connection Quality, "in press, paper accepted for XVI International ScientificProfessional Symposium Infoteh-Jahorina 2017. 